\title{
Test Method \\ Experimental investigation of the effect of temperature on the strength of polyimide contact structures
}

\author{
Qinwei Ma ${ }^{\mathrm{a}}$, Hetong Liu ${ }^{\mathrm{b}}$, Hongtao Wang ${ }^{\mathrm{c}}$, Libin Sun ${ }^{\mathrm{c}}$, Shaopeng $\mathrm{Ma}^{\mathrm{a}^{*}}$ \\ ${ }^{a}$ School of Aerospace Engineering, Beijing Institute of Technology, Beijing 100081, China \\ ${ }^{b}$ China Nuclear Power Engineering Company Ltd., Beijing 100840, China \\ ${ }^{c}$ Institute of Nuclear and New Energy Technology, Tsinghua University, Beijing 100084, China
}

\begin{abstract}
A new photogrammetry based measurement technique for contact areas in line contact structures is developed. From the data collected by this technique, a method for measuring the contact strength of contact structures in high temperature applications is proposed. The contact strength of a line contact structure of polyimide at different temperatures is measured; the results show that the increase in temperature decreases the contact strength of the structure. The effect of temperature on the contact strength is studied by investigating the occurrence and evolution of yielding core within the structure at different temperatures. The decrease in the yield strength and elastic modulus of the polyimide material at high temperature induces core yielding at lower loads and eases the propagation of core yielding to the surface.
\end{abstract}

Keywords: polyimide; contact strength; temperature effect; yielding core; digital image correlation

\section{Introduction}

Because polymers are used as both structural and functional materials in some contact structures [1, 2], such as that of a cylinder or a sphere placed on a plate and subjected to a load [3], the load capacity of these structures (i.e., the contact strength) should be measured and analysed. Such polymer structures might be used in environments of different temperatures $[4,5]$, and the temperature effect on the contact strength should be investigated. Although many have studied the effect of temperature on the mechanical properties of polymer materials [6-8], very few have reported the effect of temperature on the contact strength of polymer structures.

While the strength of a material is intrinsic, the strength of a contact structure depends on the contact type and geometry of the structure [9]. For example, for the line contact structure of a cylinder with radius $R$ and length $L$ compressed onto a plate with load $P$, a rectangular contact area forms with width $2 b$. According to Hertzian contact theory [10] and previous studies [11], the corresponding average stress $\sigma_{\text {ave }}$ (where $\sigma_{\text {ave }}=P / 2 b L$ ) of the inflection point of the curve plotting $\sigma_{\text {ave }}$ vs. $b / R$ is considered to be the contact strength of the structure. Therefore, measuring $b$, which is the half-width of the rectangular contact area, is necessary to obtain the contact strength. Unfortunately, measurement of the contact area at high temperatures is very difficult, because the methods used at normal temperatures, such as indentation $[12,13]$ and the piezoelectric film method [14], fail at elevated temperatures.

In this paper, a new measurement method for contact areas based on photogrammetry is presented. The non-contact nature of the method is suitable for high-temperature measurements. Subsequently, the contact strength of a polyimide (PI) line contact structure is measured at different temperatures

\footnotetext{
* Corresponding author. Tel.: +86 1068912321

Email address: masp@bit.edu.cn (S. P. Ma)
} 
to investigate the relationship between contact strength and temperature. The mechanism underlying the effect of temperature on the contact strength is discussed using the correspondence between the yielding process of the contact structure and the $\sigma_{\text {ave }}$ Vs. $b / R$ curve.

This paper is organized as follows. A non-contact measuring method for contact areas in hightemperature applications is introduced in section 2. The contact strength of a PI structure is measured at different temperatures and the effect of the temperature on the contact strength is discussed in section 3. In section 4 , the mechanism underlying the effect of the temperature on the contact strength of PI is investigated.

\section{Non-contact measurement method of the contact strength}

\subsection{Contact strength}

Consider a line contact structure comprising a cylinder with radius $R$ and length $L$, subjected to a linear load $P$, as shown in Fig. 1a. According to Hertzian contact theory, the global mechanical behaviour of the structure during the elastic stage [10] can be expressed as a linear relationship such that

$$
\sigma_{\text {ave }}=E^{*} \varepsilon^{*},
$$

where the average stress $\sigma_{\text {ave }}$ is given by

$$
\sigma_{a v e}=\frac{P}{2 b L}
$$

the equivalent strain $\varepsilon^{*}$ is given by

$$
\varepsilon^{*}=\frac{b}{R}
$$

and the equivalent elastic modulus $E^{*}$ of the line contact structure is given by

$$
E^{*}=\frac{\pi}{16\left(1-v^{2}\right)} E,
$$

where the parameters $E$ and $v$ are the elastic modulus and Poisson's ratio of the material, respectively, and $b$ is half the contact width (abbreviated as HCW hereafter).

\section{[Figure 1]}

An experimental investigation conducted by Ma et al. [11] showed that a certain area in the contact structure began to yield following the elastic stage, while the global behaviour remained linear for a yielding area that is not large enough. As shown in Fig. 1b, the inflection point of the relationship between $\sigma_{\text {ave }}$ and $b / R$ corresponds to the point at which the yielding area propagates to the surface of the structure, thereby indicating the characteristic yielding state of the structure. Therefore, the average stress at the inflection point of the relationship expressed in Eq. (1) can be regarded as the contact strength of the contact structure. Clearly, the measurement of HCW is necessary to evaluate the contact strength, but this is generally difficult, especially in high-temperature environments. To investigate the contact strength of PI structures at different temperatures, a non-contact method to measure the HCW must be developed.

\subsection{Contact area measurement method based on photogrammetry}

To measure the contact area at high temperatures, a measurement method based on photogrammetry was developed. As shown in Fig. 2a, a light source was placed at the back of the contact structure, where a specially designed aperture was used to control the amount of light projected on the scattering screen. This then generates a uniform light intensity behind the structure. A digital CCD 
camera in front of the structure was then used to obtain an image of the contact area. Before the experiment, the light and camera were carefully adjusted to obtain photographs with very high contrast. After the experiment, the captured images were processed using edge detection algorithms (as shown in Fig. 2b), from which the HCW was calculated as half of the distance between the two intersecting points of the two edges $\mathrm{A}$ and $\mathrm{B}$ of the contact structure.

[Figure 2]

To verify the proposed method, a contact experiment using a graphite cylinder and a graphite plate under linear loading was conducted, as shown in Fig. 3a. The experiment was performed at room temperature to compare the HCW results obtained from both the traditional pressure film method and the developed photogrammetry method. The measurement was conducted via a stepped loading mode. For each step, a piece of unused pressure film (Fuji, LLLW, Japan) was placed between the cylinder and the plate, the structure was loaded to a particular level and an image was captured. The structure was then unloaded and the pressure film was removed for data analysis. Examples of the original data obtained by the pressure film method and that by the photogrammetry method are shown in Fig. 3b. Two sets of HCW data could be obtained with each measurement by processing the photogrammetry images and directly measuring the rectangles imprinted on the pressure film. The agreement between the results by these two methods, shown in Fig. 3c, verifies the effectiveness of the photogrammetry method.

[Figure 3]

\section{Contact strength of a PI structure at different temperatures}

Based on the non-contact HCW measurement technique proposed above, the contact strength of PI at different temperatures was experimentally investigated. The experimental setup is shown in Fig. $4 \mathrm{a}$; the dimensions of the PI cylinder and plate used in this experiment are shown in Fig. $4 \mathrm{~b}$. The light source used was a temperature-resistant lamp with a scattering screen composed of hightemperature ceramic fibres. The cylinder was compressed using a MTS 810 testing machine (MTS Systems Corp., USA) in displacement control mode at a speed of $0.1 \mathrm{~mm} / \mathrm{min}$. A MTS 651 environment chamber (MTS Systems Corp., USA) was used for the high-temperature environment, and a CCD camera (IMPERX 16M-3L, IMPERX Inc., $4872 \times 3248$ pixels, USA) with a frame rate of $0.2 \mathrm{fps}$ was used to obtain the images. The experimental setup is shown in Fig. 4c, and one captured photograph of the specimen is shown in Fig. 4d.

[Figure 4]

The measurement was conducted at three different temperatures of 20,100 , and $200^{\circ} \mathrm{C}$. At each temperature, the HCW was obtained as the load was increased; the average stress and equivalent strain were subsequently calculated from the load. The curves of $\sigma_{\text {ave }} \mathrm{vs} . b / R$ for the contact structure at different temperatures are shown in Fig. 5a.

As described in section 2.1, the contact strength of PI at different temperatures can be evaluated from the measured $\sigma_{\text {ave }}$ vs. $b / R$ curves. The measured contact strength at the three temperatures is shown in Fig. 5b, which demonstrates that the temperature apparently influences the PI contact structure, with decreases in the contact strength as the temperature is increased.

[Figure 5]

\section{Mechanism of temperature effect on the contact strength of PI contact structure}

The mechanism underlying the temperature effect observed on the contact strength of the PI contact structure was analysed. As described in section 2.1, the mechanical parameters of the structure can affect the global curve of $\sigma_{\text {ave }}$ vs. $b / R$, as well as the local deformation behaviour. Temperature is 
known to be an important factor affecting the mechanical parameters of polymers [6-8]; therefore, the mechanism underlying the change in contact strength at different temperatures was analysed by determining the mechanical parameters and analysing the local deformation behaviour of the PI material at different temperatures.

The specimen used to investigate the mechanical behaviour of PI was manufactured as a square column measuring $20 \times 20 \times 50 \mathrm{~mm}$. The front surface of the column was coated with paint speckles, as shown in Fig. 6a, using a high-temperature spray paint. The loading machine, CCD camera and environment chamber used here were the same as those used in section 3 . The mechanical parameters were tested by loading the specimen in the environment chamber at temperatures of 20,100 , and $200^{\circ} \mathrm{C}$. During the experiment, the specimen was loaded and images of the speckles were captured. From these images, the displacement field corresponding to a certain load was obtained by analysing the speckle images using the digital image correlation method [15]. Typical longitudinal and vertical displacement fields from the experiment at $20^{\circ} \mathrm{C}$ are shown in Fig. $6 \mathrm{~b}$ and $\mathrm{c}$, respectively. By fitting the displacement fields to a polynomial function, the longitudinal and vertical strains are obtained [16], with which the stress-strain curve of the material is produced, as shown in Fig. 6d. From the derived curve, the elastic modulus E, Poisson's ratio $v$, and yield strength $\sigma_{s}$ were obtained. Because the maximum shear stress criterion is typically used to characterize the mechanical behaviour of PI [11], the shear yield strength $\tau_{s}$ was calculated as $\sigma_{s} / 2$, as listed in Table. 1. Both $E$ and $\sigma_{s}$ decrease with increases in temperature.

\section{[Figure 6]}

Table 1 Measured mechanical parameters of PI at different temperatures.

\begin{tabular}{ccccc}
\hline $\begin{array}{c}\text { Temperature } \\
\left({ }^{\circ} \mathrm{C}\right)\end{array}$ & $\begin{array}{c}\text { Elastic modulus } \\
E(\mathrm{GPa})\end{array}$ & $\begin{array}{c}\text { Poisson's } \\
\text { ratio } v\end{array}$ & $\begin{array}{c}\text { Yield strength } \sigma_{s} \\
(\mathrm{MPa})\end{array}$ & $\begin{array}{c}\text { Shear yield strength } \\
\tau_{\mathrm{s}}(\mathrm{MPa})\end{array}$ \\
\hline 20 & 3.51 & 0.42 & 62 & 31 \\
\hline 100 & 3.15 & 0.43 & 52 & 26 \\
\hline 200 & 2.96 & 0.43 & 50 & 25 \\
\hline
\end{tabular}

Based on the variations in the mechanical behaviour of PI at different temperatures, the decreased contact strength can be described qualitatively. As mentioned in section 2.1, the inflection of the curve of $\sigma_{\text {ave }}$ Vs. $b / R$ (i.e., the global yielding of the contact structure) comprises two key stages of the occurrence of core yielding within the structure, and the propagation of this yielding to the surface. Reduced yield strengths are hypothesized to induce core yielding at reduced loads, while reduced elastic modulus may ease material deformation and thereby the propagation of the core yielding. Therefore, the increased temperature decreases the contact strength of the PI structure.

To verify this analysis, an experiment quantitatively measuring the local yielding process of the structure was conducted. The experimental setup and specimen, shown in Fig. 7a and b, respectively, were the same as those in the experiment in section 3 . The front surface of the specimen was similarly painted with speckles using high-temperature spray paint, as shown in Fig. 7c. A CCD camera (AVT PIKE F-421B, AVT Inc., $2048 \times 2048$ pixels, Germany) with a frame rate of 1 fps was used to obtain the images. During loading, the evolution of the deformation fields was measured using the digital image correlation method, with typical displacement and strain fields as shown in Fig. $7 \mathrm{~d}-\mathrm{h}$.

\section{[Figure 7]}

The maximum shear strain field $\left(\gamma_{\max }\right)$, which can be calculated from the measured strain fields, was used to express the yielding process considered by the Tresca yield criterion. Five typical $\gamma_{\max }$ fields under different loads at each temperature are shown in Fig. 8. The PI cylinder yields beginning at the point when $\tau_{\max }$ exceeds the Tresca yielding stress $\tau_{s}$, with increasing load, whereupon core yielding occurs. To streamline the following analysis, the areas in Fig. 8 where $\gamma_{\text {max }}$ exceeds Tresca yielding strain $\gamma_{s}=2(1+v) \tau_{s} / E$ are coloured white to represent core yielding . 
The first column of the $\gamma_{\max }$ fields, presented as Fig. 8a, f, and k, shows the elastic behaviour of the structure at a loading level of $35.5 \mathrm{kN}$. The strain value in the concentration area is larger at higher temperatures, which is reasonable because of the decreased elastic modulus existing at higher temperatures. The second column of images (Fig. 8b, g, and 1) shows the occurrence of core yielding at the three temperatures. Consistent with the analysis above, core yielding occurs at lower loads for higher temperatures. The solid line in Fig. 9 plots the load at which yielding occurs $\left(F_{\text {occ }}\right)$ for the different temperatures; $F_{\text {occ }}$ decreases with increasing core temperature, implying that core yielding occurs more easily at higher temperatures. The third, fourth, and fifth columns of images show the progression of core yielding propagation at the three different temperatures. The fields in the third column (Fig. 8c, h, and $\mathrm{m}$ ) show that core yielding is propagated with increased load, and reached the surface of the cylinder (corresponding to the inflection point of the $\sigma_{\text {ave }} \mathrm{Vs} . b / R$ curve) first at $200^{\circ} \mathrm{C}$ with a load increment of $1.6 \mathrm{kN}$. However, under the same load increment, core yielding does not reach the surface at $20^{\circ} \mathrm{C}$ and $100^{\circ} \mathrm{C}$. The fields in the fourth (Fig. $8 \mathrm{~d}$, i, and n) and fifth (Fig. 8e, j, and o) columns show the core yielding reaching the cylinder surfaces at $100^{\circ} \mathrm{C}$ (load increment: $0.6 \mathrm{kN}$ ) and $20^{\circ} \mathrm{C}$ (load increment: $1.3 \mathrm{kN}$ ), respectively. Under the same load increment, core yielding does not reach the surface at a lower temperature; meanwhile, the structure fails at a higher temperature. The three fields within the dashed lines (Fig. 8m, i, and e) indicate, crucially, that core yielding reaches the surface under different loads at different temperatures. The corresponding load $F_{\text {sur }}$ at which core yielding reaches the surface is plotted in Fig. 9 using a dashed line. The increment between $F_{\text {sur }}$ and $F_{\text {occ }}$ is decreased at increased temperatures, which means that the propagation of core yielding to the surface is easier at increased temperatures.

\section{[Figure 8]}

\section{[Figure 9]}

As analysed and revealed by the experiment, increased temperatures decrease the yield strength and elastic modulus of PI, which eases the occurrence and propagation of core yielding within the contact structure. By assuming that the yield mechanism of the contact structure proceeds as mentioned in section 2.1, the decreased contact strength with increased temperature is explained.

\section{Conclusions and discussion}

A new measurement method for the contact area of load-bearing contact structures, based on photogrammetry, is presented. The non-contact nature of the method is suitable for hightemperature measurement. Moreover, because the influence of temperature on the mechanical properties of polymer materials is universal, the influence of temperature on the contact strength can be considered using the analysis presented here when other polymers are used in hightemperature applications.

Experimental results demonstrated that the contact strength of a PI structure decreases with the increase in temperature because of the influence of temperature on the material's mechanical properties. First, the material yields more easily with increasing temperature, thereby allowing yielding core at smaller loads. Second, the constraint ability of the material is weakened by the decrease of the elastic modulus with increased temperature; therefore, core yielding propagates more easily to the structure's surface.

\section{Figure captions}

Fig. 1. Cylindrical line contact structure and its yielding procedure: $(a)$ schematic of the contact structure; $(b)$ deformation and yielding progression of the cylinder [11]. 
Fig. 2. Schematics of the photogrammetry-based HCW measurement method: (a) experimental setup; (b) data processing.

Fig. 3. Verification of the photogrammetry-based HCW measurement method: $(a)$ experimental arrangement; $(b)$ original data obtained by the pressure film method (upper) and photogrammetry method (lower); (c) comparison of the measurement results by the two methods.

Fig. 4. Experimental contact strength measurement at high temperature: $(a)$ schematic of the experimental setup; $(b)$ dimensions of the PI cylinder and plate; (c) photograph of the experiment arrangement; $(d)$ image of the specimen.

Fig. 5. Contact strength of PI contact structure at different temperatures: $(a) \sigma_{\text {ave }}$ vs. $b / R$ curves at different temperatures; $(b)$ variation in contact strength with temperature.

Fig. 6. Measurement of the mechanical behaviour of PI material using digital image correlation: (a) speckle image obtained by CCD camera; $(b)$ longitudinal displacement field in the $x$ direction; $(c)$ vertical displacement field in the $y$ direction; $(d)$ stress-strain curve of PI at $20^{\circ} \mathrm{C}$.

Fig. 7. Measurement of the yielding process of the PI structure at different temperatures: $(a)$ experimental arrangement; $(b)$ specimen; $(c)$ example of original data obtained by the CCD camera; $(d-h)$ displacement fields in the $x$ and $y$ directions; $(f-g)$ strain fields in the $x$ and $y$ directions; $(h)$ shear strain field.

Fig. 8. $\gamma_{\max }$ fields of the contact structures at $(a-e) 20^{\circ} \mathrm{C},(f-j) 100^{\circ} \mathrm{C}$, and $(k-o) 200^{\circ} \mathrm{C}$.

Fig. 9. Variations in load corresponding to yielding core occurrence of the yielding core $\left(F_{\text {occ }}\right)$ and that corresponding to yielding core reaching the surface $\left(F_{\text {sur }}\right)$ at three different temperatures.

\section{Acknowledgment}

Funding: This research was supported by the National Science Foundation of China (Nos. 11372038 and 11402023).

\section{References}

[1] A.K. Ray, S. Chattopadhyaya, A.K. Mukherjee. Polymer applications in pulp and papermaking - A review. In: Proceedings of AIChE Annual Meeting, 2012.

[2] J. Qin, H. Ding, X. Wang, M.J. Xie, Z.L. Yu. Blending LLDPE and ground rubber tires. PolymPlastics Technol Eng 2008;47(2):199-202.

[3] P.H. Pinchbeck. A review of plastic bearings. Wear 1962;5(2):85-113.

[4] M. Iwata, A. Ohnishi, H. Hirosawa, F. Tohyama. Measurement and evaluation of thermal control material with polyimide for space use. J Spacecr Rockets 2001;38(4):504-509.

[5] S.M. Homam, S.A. Sheikh. Fiber-reinforced polymers exposed to nuclear power plant environment. J Compos Constr 2013;17(6):04013007. doi: 10.1061/(ASCE)CC.19435614.0000396

[6] C.W. Paul, Mechanical properties of polypivalolactone: effects of thermal history and solvent exposure, Journal of Applied Polymer Science, 36 (3) (1988) 675-689.

[7] H. Aglan, Z. Qian, D. Mitra-Majumdar. The effect of temperature on the critical failure properties of advanced polymer composites. Polym Test 1992;11(3):169-184. 
[8] D. Evans, J.T. Morgan. Low temperature mechanical and thermal properties of liquid crystal polymers. Cryogenics 1991;31(4):220-222.

[9] J.E. Shigley, C.R. Mischke, T.H. Brown. Standard handbook of machine design. 3rd ed. McGraw Hill. 2004

[10] K.L. Johnson. Contact mechanics. Cambridge University Press. 1987

[11] S.P. Ma, J.Z. Pang, Q.W. Ma, X. Wang, H.T. Wang. Experimental investigation of the yielding process of a ductile polycarbonate cylinder subjected to line loading using digital image correlation. Polym Test 2013;32(3):461-467.

[12] H. Kim, C. Ulan-Kvitberg, C. Daley. Evaluation of spatial pressure distribution during icestructure interaction using pressure indicating film. Int J Nav Archit Ocean Eng 2014;6(3):578597.

[13] M.L. Harris, P. Morberg, W.J.M. Bruce, W.R. Walsh. An improved method for measuring tibiofemoral contact in total knee arthroplasty: a comparison of K-scan sensor and Fuji film. J Biomech 1999; 32:951-958.

[14] R.L. Sakaguchi, B.S. Wenande, R. DeLong, G.C. Anderson, W.H. Douglas. A piezoelectric film transducer for dental occlusal analysis. Clin Mater 1992;10(3):145-151.

[15] S.P. Ma, Z.L. Zhao, X. Wang. Mesh-based digital image correlation method using higher order isoparametric elements. J Strain Anal Eng Des 2012;47(3):163-175.

[16] H.W. Schreier, M.A. Sutton, J. Orteu. Image correlation for shape, motion and deformation measurements: basic concepts, theory and applications. Springer. 2009 

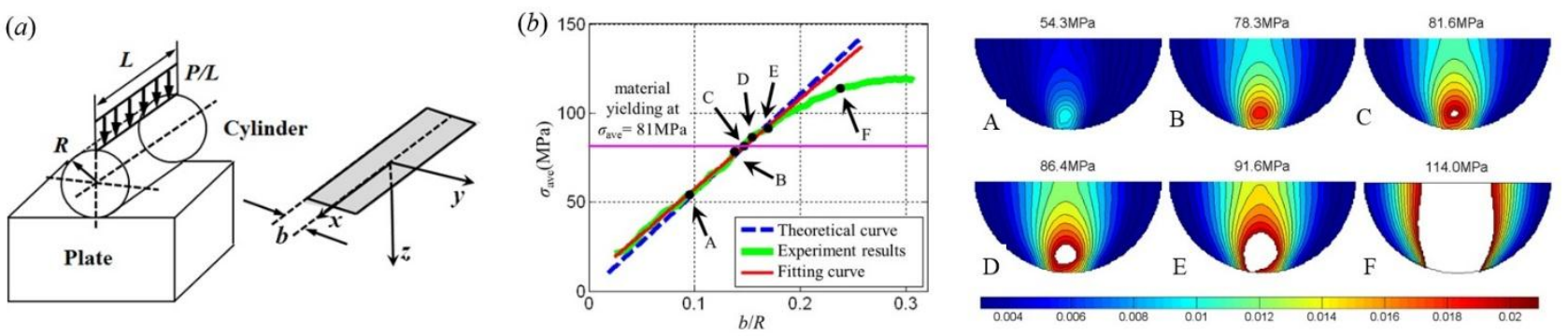
(a)

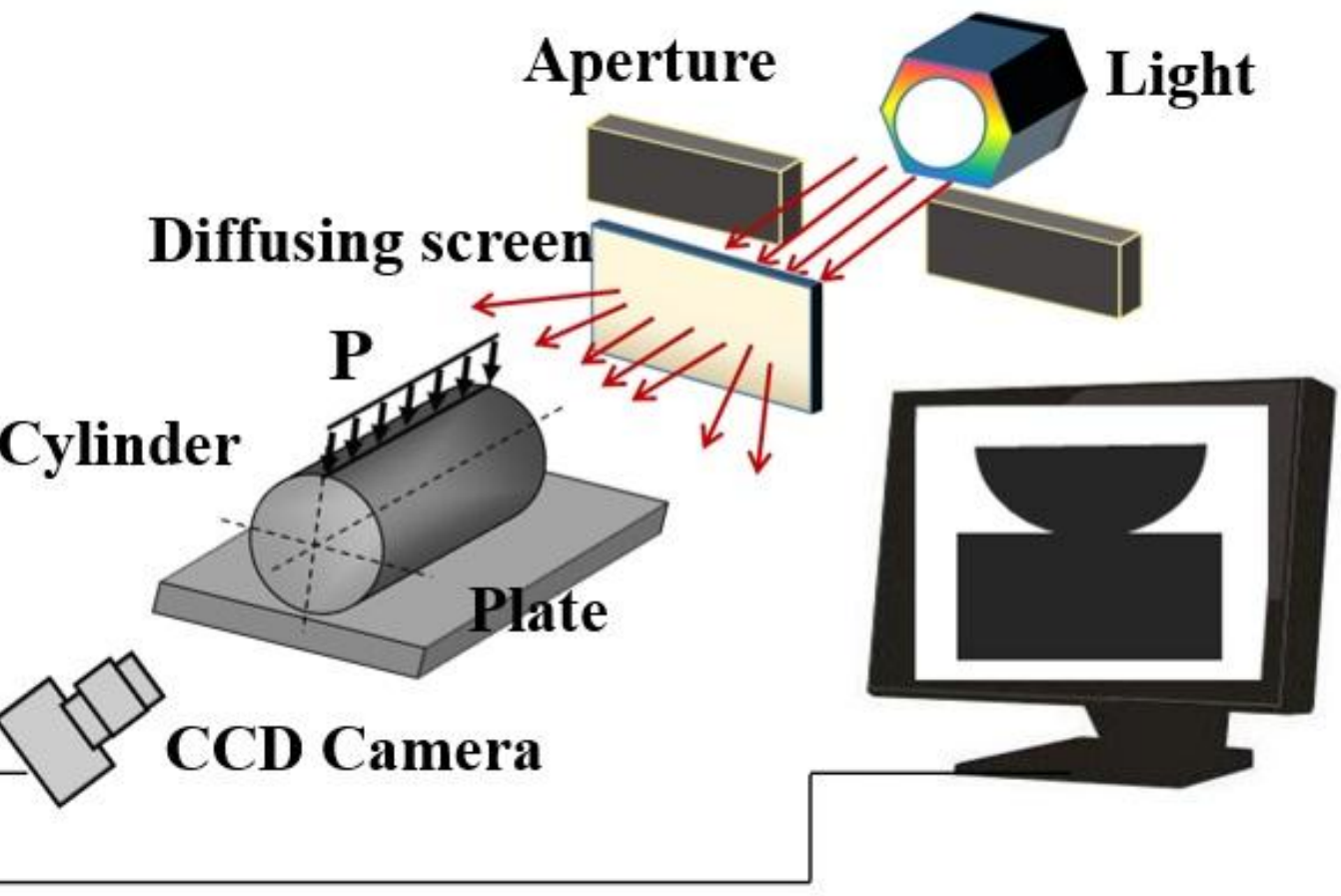

(b)
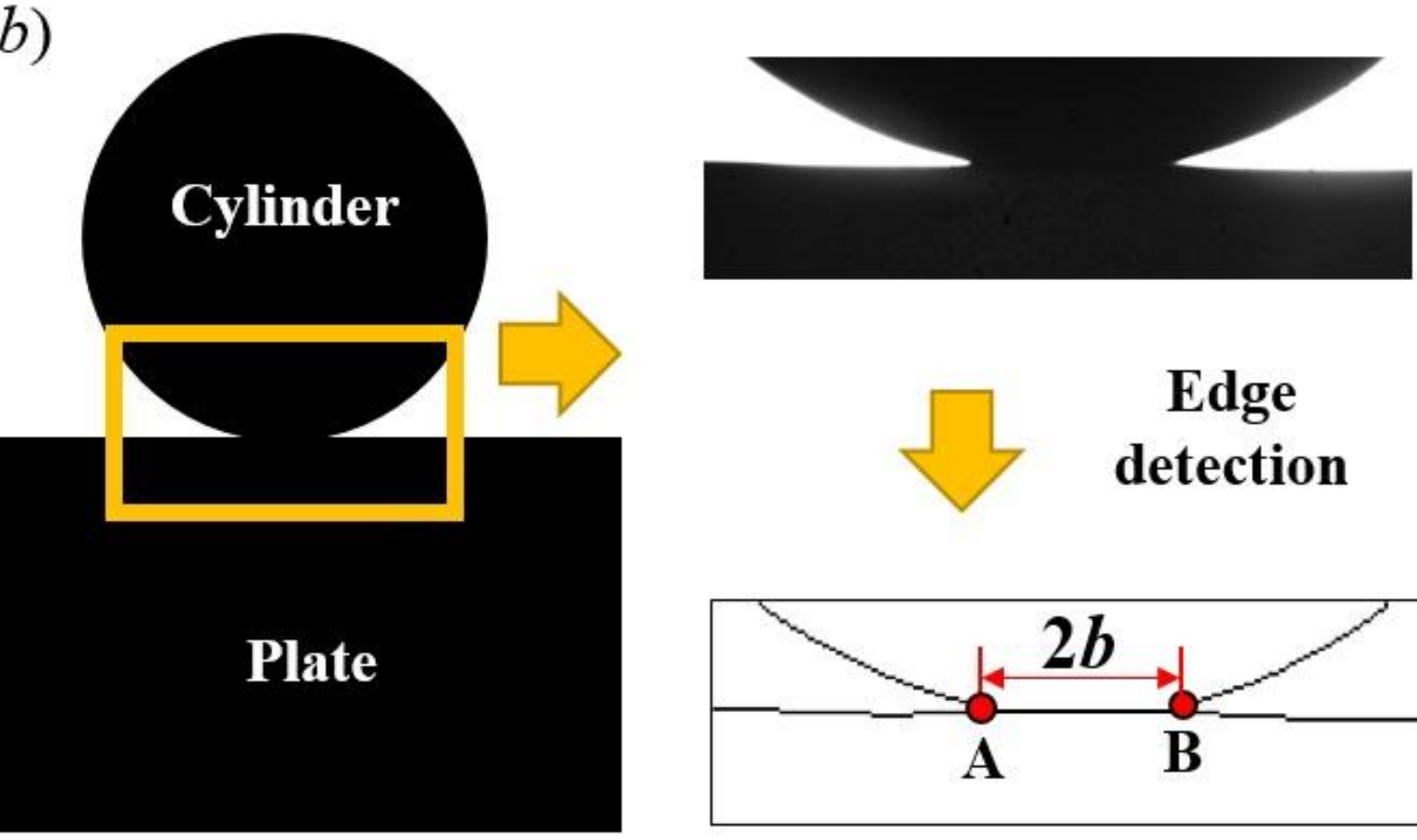

Edge

detection

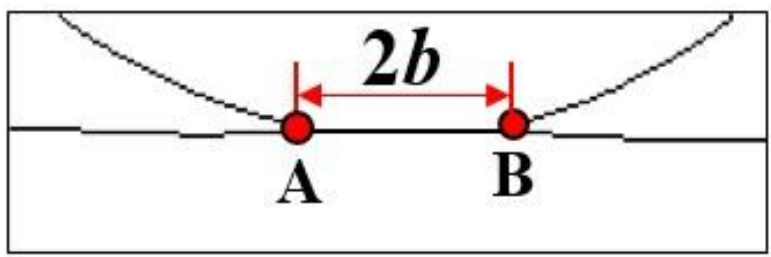



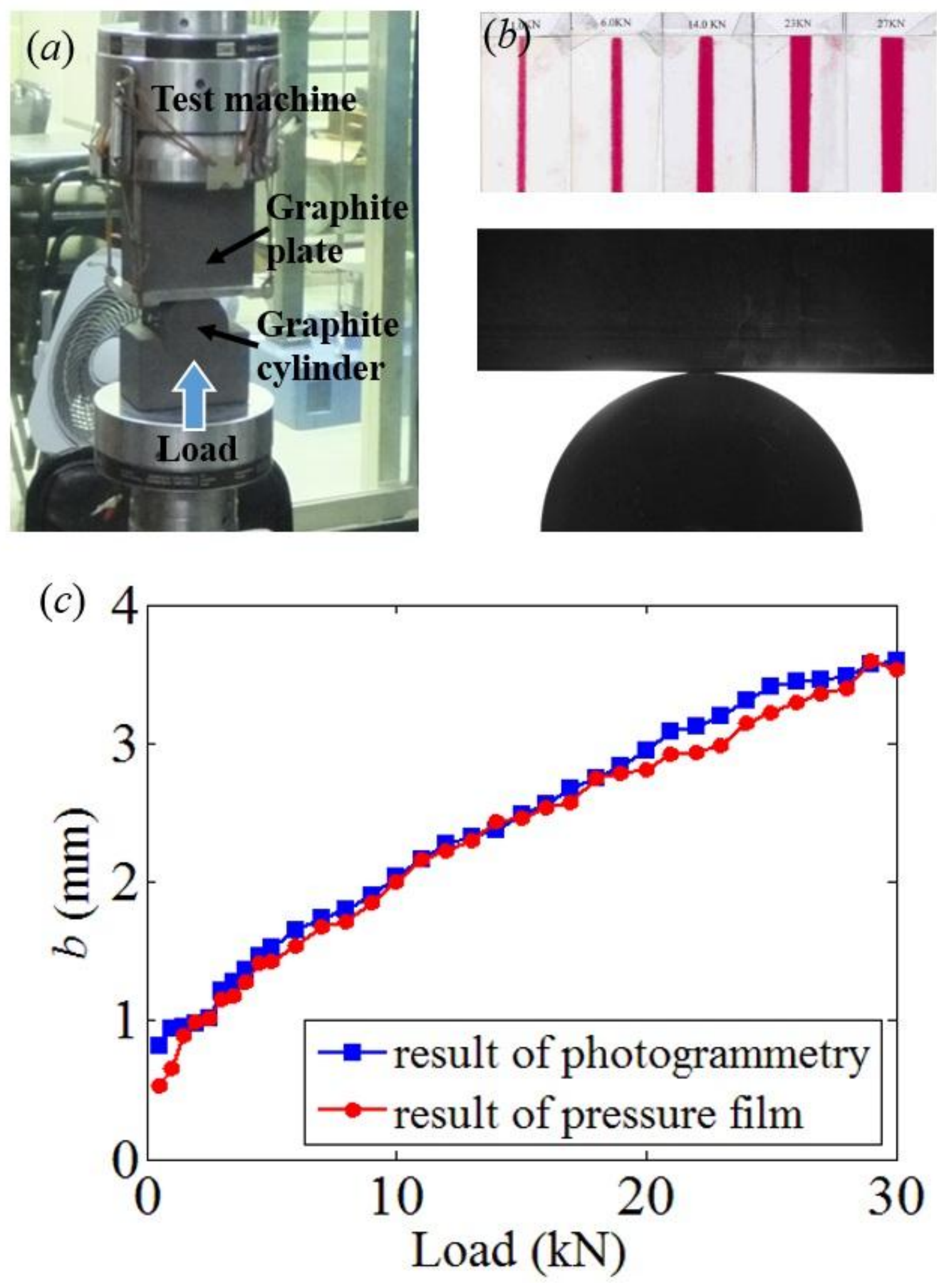

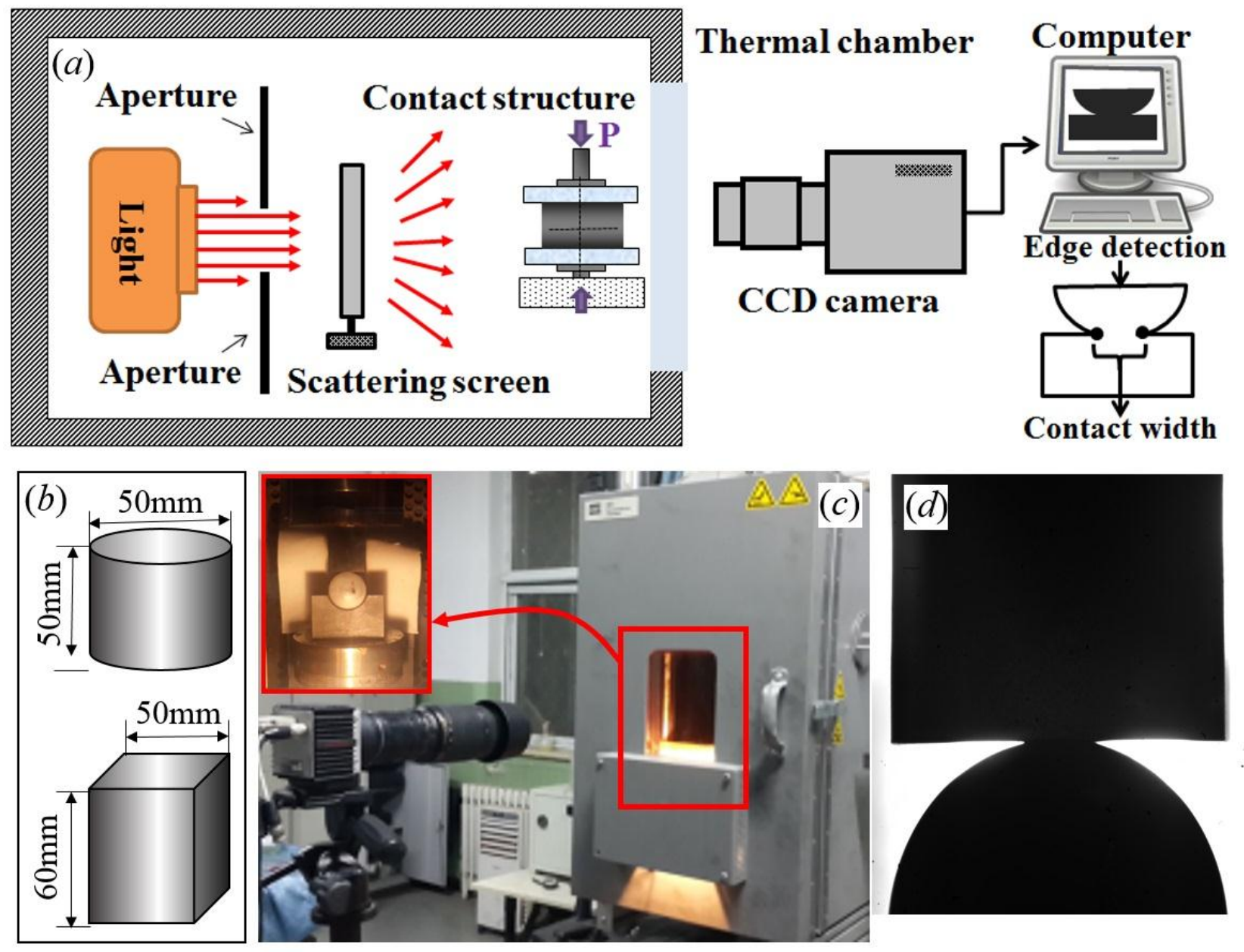

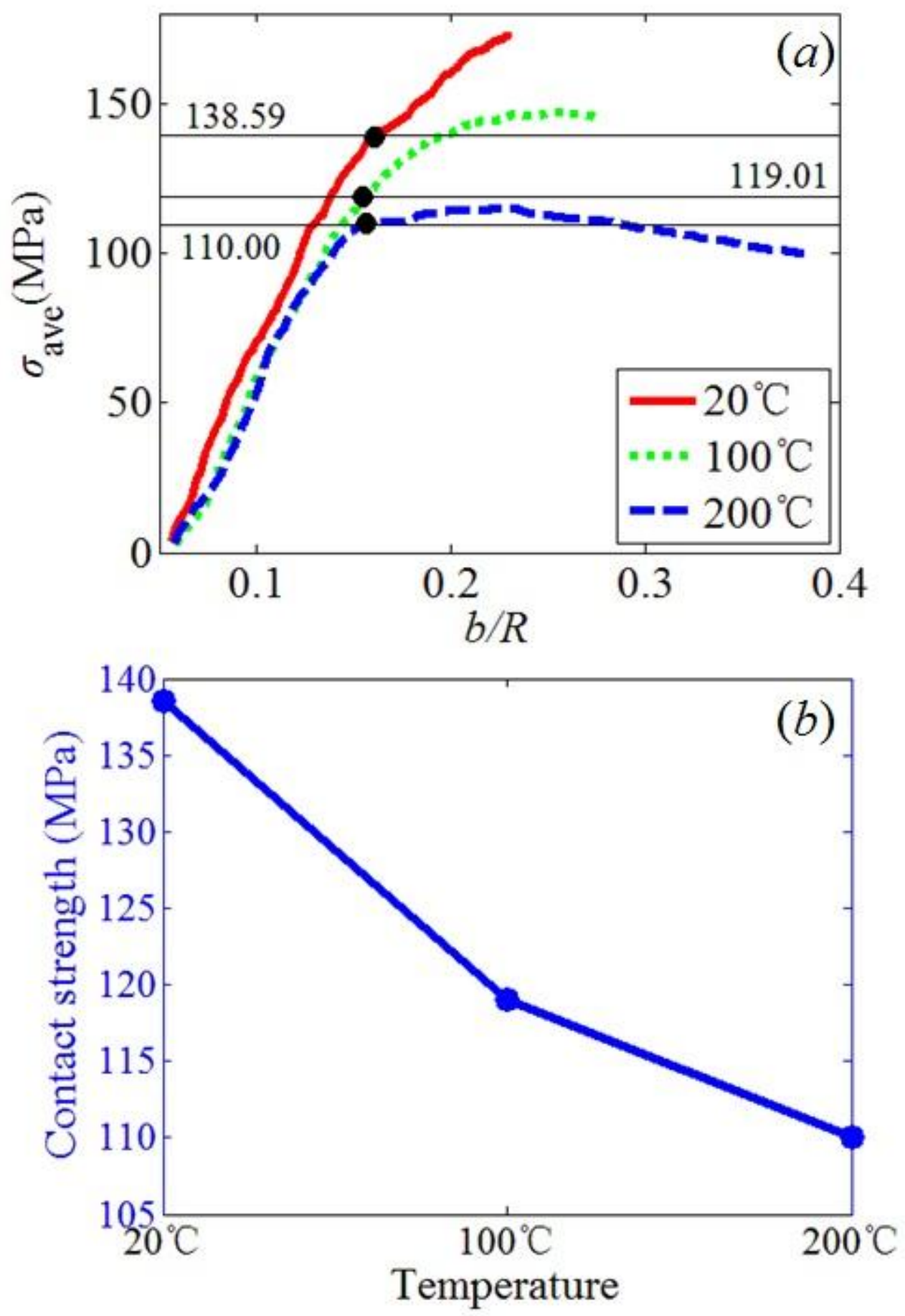

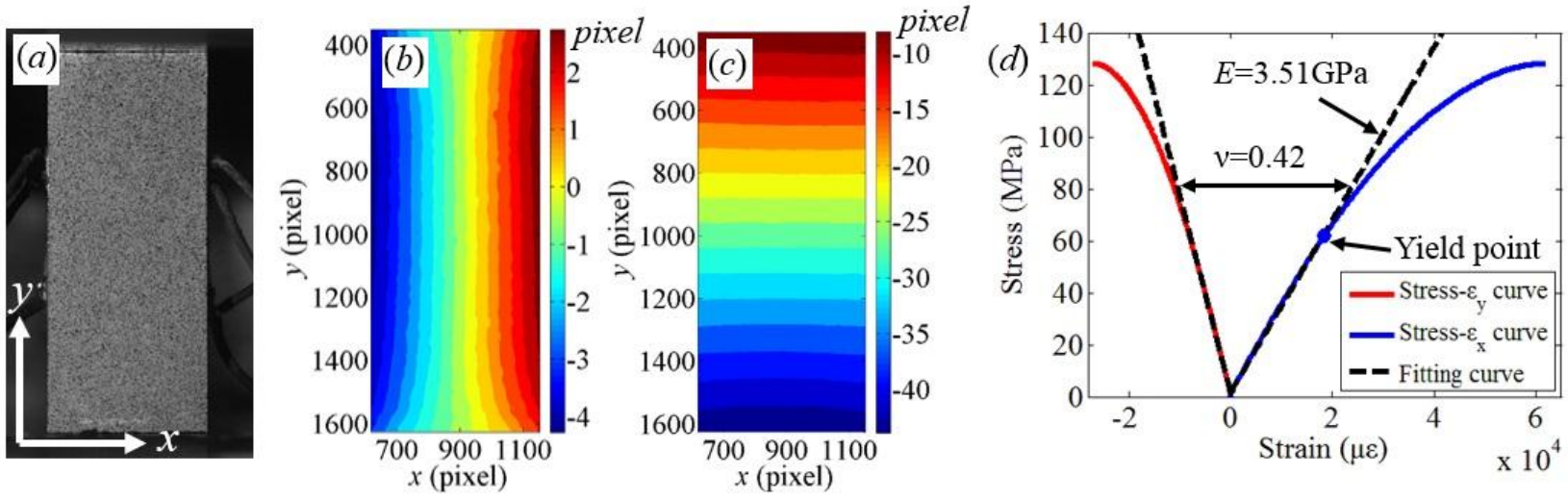


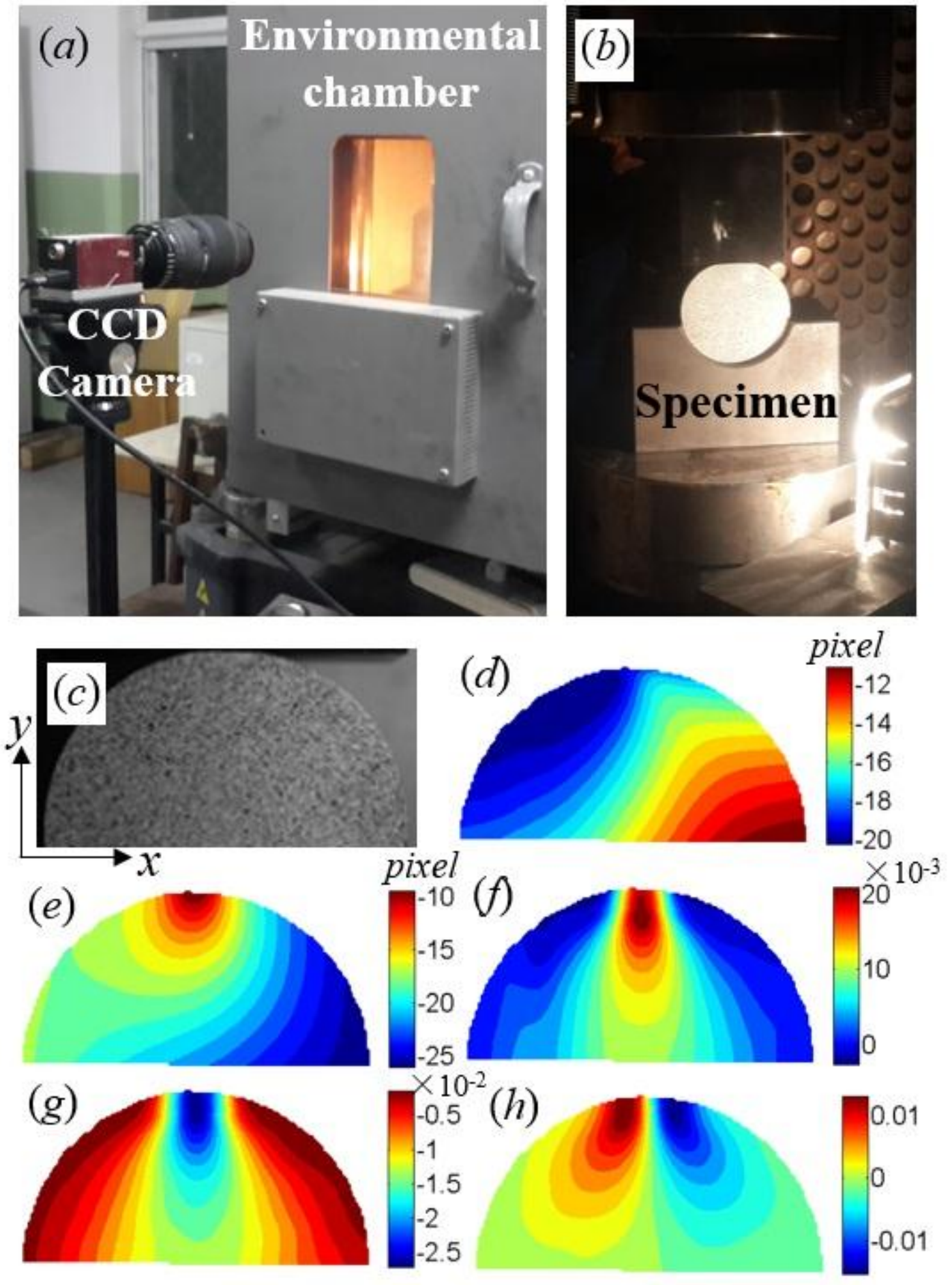




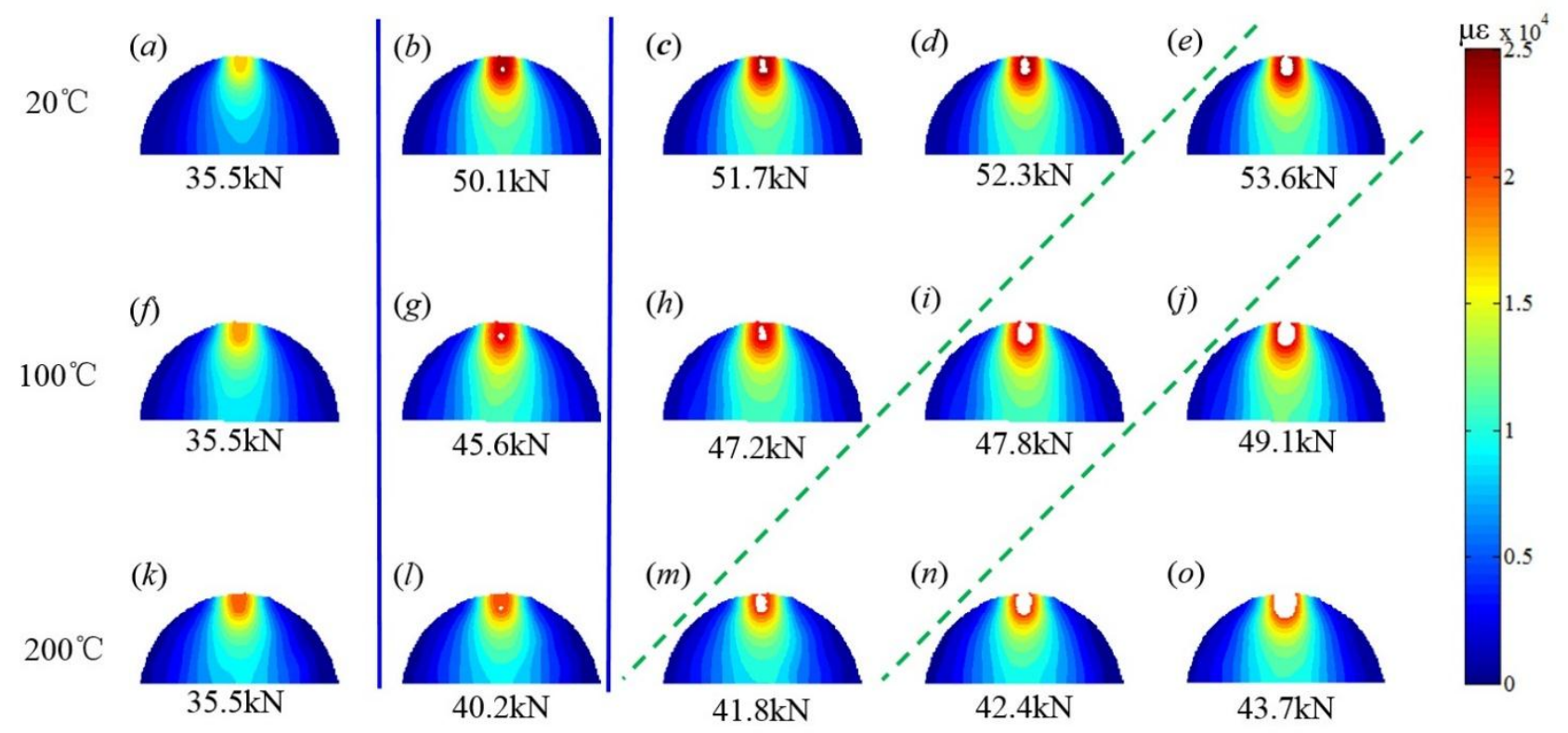




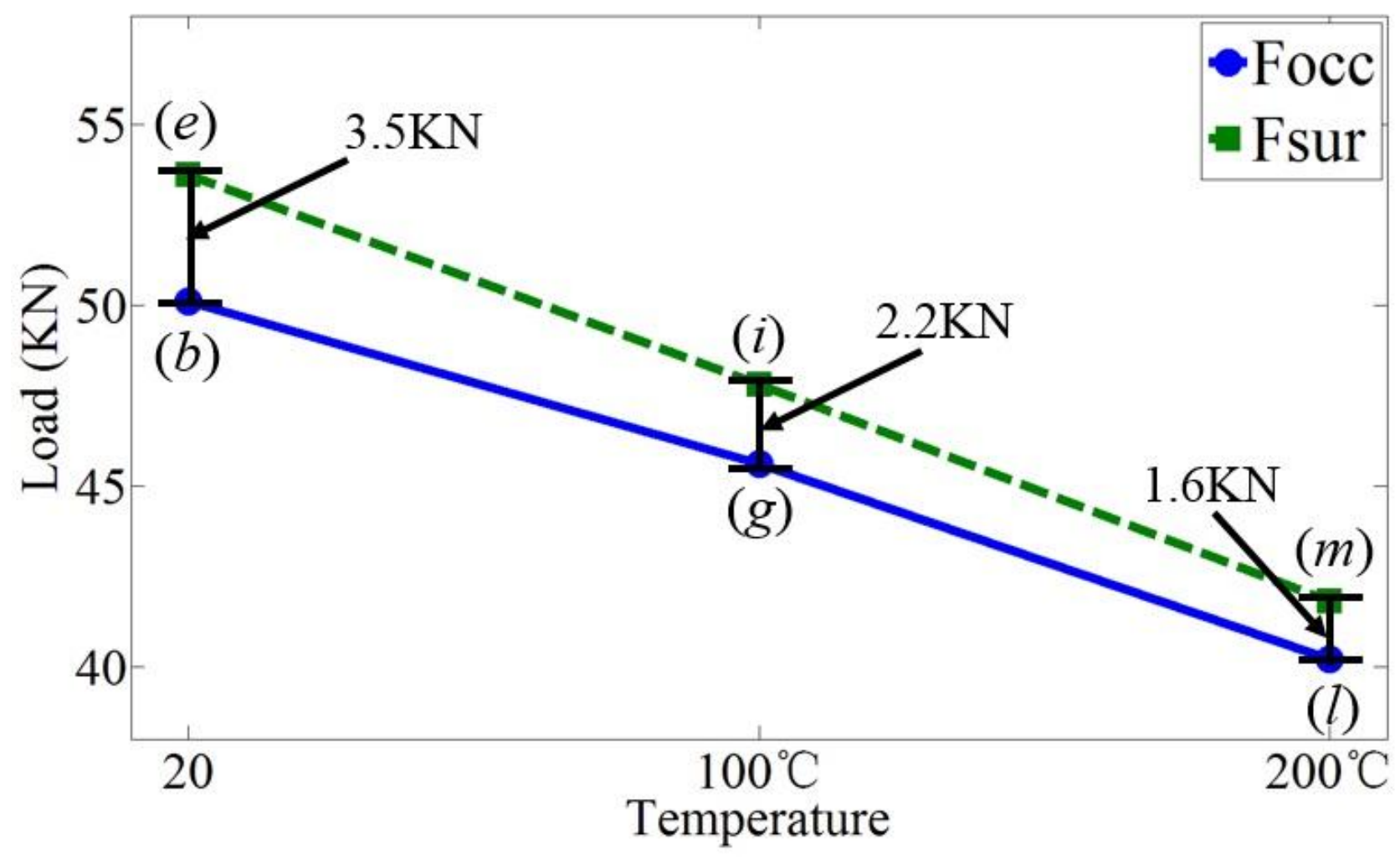

